\title{
Angular distributions of photoelectrons and interatomic-Coulombic-decay electrons from helium dimers: Strong dependence on the internuclear distance
}

\author{
T. Havermeier, ${ }^{1}$ K. Kreidi, ${ }^{1}$ R. Wallauer, ${ }^{1}$ S. Voss, ${ }^{1}$ M. Schöffler, ${ }^{1}$ S. Schössler, ${ }^{1}$ L. Foucar, ${ }^{1}$ N. Neumann, ${ }^{1}$ J. Titze, ${ }^{1}$ H. Sann, ${ }^{1}$ \\ M. Kühnel, ${ }^{1}$ J. Voigtsberger, ${ }^{1}$ N. Sisourat,${ }^{2}$ W. Schöllkopf, ${ }^{3}$ H. Schmidt-Böcking, ${ }^{1}$ R. E. Grisenti, ${ }^{1,4}$ R. Dörner, ${ }^{1, *}$ and T. Jahnke ${ }^{1}$ \\ ${ }^{1}$ Institut für Kernphysik, J. W. Goethe Universität, Max-von-Laue-Strasse 1, D-60438 Frankfurt, Germany \\ ${ }^{2}$ Institut für physikalische Chemie, Universität Heidelberg, Im Neuenheimer Feld 229, D-69120 Heidelberg, Germany \\ ${ }^{3}$ Fritz-Haber-Institut der Max-Planck-Gesellschaft, Faradayweg 4-6, D-14195 Berlin, Germany \\ ${ }^{4}$ GSI Helmholtzzentrum für Schwerionenforschung GmbH Planckstrasse 1, D-64291 Darmstadt, Germany
}

(Received 28 September 2010; published 10 December 2010)

\begin{abstract}
In the present paper, we show that the absorption of a single photon can singly ionize both atoms of a helium dimer $\left(\mathrm{He}_{2}\right)$ : ionization with simultaneous excitation of one atom followed by de-excitation via interatomic Coulombic decay leads to the ejection of an electron from each of the the two atoms of the dimer. Using the Cold Target Recoil Ion Momentum Spectroscopy technique (COLTRIMS), we obtained angular distributions of these electrons in the laboratory frame and the molecular frame. We observe a pronounced variation of these distributions for different regions of kinetic-energy releases of the ions.
\end{abstract}

DOI: 10.1103/PhysRevA.82.063405

PACS number(s): 33.80.Eh, 36.40.Mr, 33.20.Tp

\section{INTRODUCTION}

Interatomic Coulombic decay (ICD) was first predicted by Cederbaum and coworkers for molecular clusters [1]. It has been observed experimentally in various van der Waals clusters consisting of $\mathrm{Ne}, \mathrm{Ar}$, and $\mathrm{Xe}$ atoms (see, e.g., [2-5]) and even water molecules [6,7]. In contrast to these many-electron systems observed so far, the helium dimer $\left(\mathrm{He}_{2}\right)$ has only four electrons. It is the most fundamental system in which ICD can occur.

In a shakeup process, we ionize and excite one atom of a helium dimer with a single photon $h v$ from a synchrotron source. An intermediate excited dimer ion $\mathrm{He}_{2}{ }^{+*}$ is created:

$$
\mathrm{He}_{2}+h v \rightarrow \mathrm{He}_{2}^{+*}+e_{\text {photo }}^{-}
$$

As ICD occurs, these excited states [e.g., $\mathrm{He}^{+*}(2 p)$ ] can de-excite by transferring the excitation energy via Coulomb interaction to the neutral neighbor, at which it causes the ejection of an ICD electron. This interaction can also be considered as the exchange of a virtual photon [8,9]:

$$
\mathrm{He}_{2}{ }^{+*} \rightarrow \mathrm{He}^{+}+\mathrm{He}^{+}+e_{\mathrm{ICD}}^{-}
$$

In a terminal step, the two ions in the final state are driven apart by the repulsive Coulomb potential in a Coulomb explosion. To good approximation, the kinetic energy release (KER), which is the kinetic energy of the ions in the centerof-mass frame, is inversely proportional to the internuclear distance at the instant of ICD.

In a previous Letter [10], we have demonstrated experimentally the existence of ICD in the helium dimer and focused on the distribution of the KER. It exhibits an oscillatory structure, which results from the vibrational structure of the intermediate $\mathrm{He}_{2}{ }^{*+}$ state [11]. We also found a direct photo double-ionization channel of $\mathrm{He}_{2}$, which, however, is much weaker than ICD [12]. In the present paper, we show results from the same experimental run as [10]. We analyze the angular

*Doerner@atom.uni-frankfurt.de distribution of the photoelectrons in the laboratory frame and the ICD electrons in the body fixed frame of the molecule. The observed angular distributions are much richer in structure than all ICD electron angular distributions reported before for more complex systems [13-16].

\section{EXPERIMENT}

The experiment was performed at beamline UE112PGM2 of the BESSY synchrotron. We used the Cold Target Recoil Ion Momentum Spectroscopy technique (COLTRIMS) to measure the momentum vectors of ions and electrons created in the photo reaction in coincidence [17-19]. By driving helium gas through a $5-\mu \mathrm{m}$ nozzle into vacuum $\left(1.2 \times 10^{-4}\right.$ mbar), we created a supersonic gas jet that contained helium dimers. The nozzle was cooled to $18 \mathrm{~K}$ by a continuous flow cryostat while the driving pressure was set to 1.8 bar. With these conditions, one can achieve a dimer fraction of $1-2 \%$ in the gas jet $[20,21]$. The target density is in the range of $10^{11}$ atoms $/ \mathrm{cm}^{3}$, which corresponds to a statistical distance of more than $1 \mu \mathrm{m}$ between the particles. Thus, reactions between (unbound) helium atoms can be neglected. The gas beam enters a scattering chamber through a $0.3-\mathrm{mm}$ skimmer, where it is crossed with a linearly polarized photon beam from the synchrotron. The interaction region is located inside a COLTRIMS spectrometer. Charged fragments from this region are guided by homogenous electric and magnetic fields $(12.2 \mathrm{~V} / \mathrm{cm}, 10.3 \mathrm{G})$ onto two position- and timesensitive detectors. These detectors consist of multichannel plates equipped with a delay-line anode for position readout [22]. Electrons are passing an acceleration region $72 \mathrm{~mm}$ long, followed by a $140-\mathrm{mm}$ field-free drift region. Thus, their time of flight (TOF) in the spectrometer is between 37 and $65 \mathrm{~ns}$ at a photon energy of $68.86 \mathrm{eV}$. Ions are accelerated in the opposite direction in a section $23 \mathrm{~mm}$ long. Singly charged helium ions in the energy range observed travel for 800-1900 ns in the spectrometer before they hit the detector.

From the TOF and the position of impact on the detector, the three-dimensional (3D) momentum vector was obtained for 


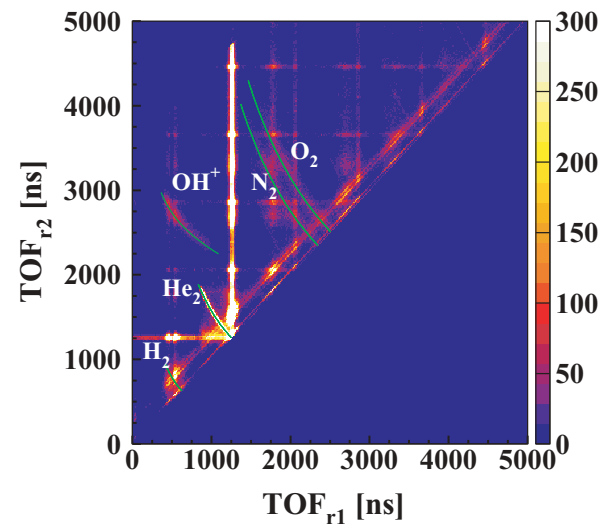

FIG. 1. (Color online) TOF in the spectrometer of two detected ions. The curved diagonals result from the Coulomb breakup of helium dimers and other molecules in the residual gas. A simulation of the spectrometer (green curves) marks the expected positions for the different charge-to-mass ratios. The vertical and horizontal features results from random coincidences. The double diagonal is an electronic artifact of the detector.

each particle. In addition, we obtain the charge-to-mass ratio from the TOF. A Coulomb breakup of a dimer into two $\mathrm{He}^{+}$ ions creates a clear signature in the data, since the ions have momenta which are directed oppositely. In Fig. 1, the TOF of one ion is plotted versus the TOF of a second detected ion. Whenever two ions originate from a two-particle Coulomb explosion, momentum conservation leads to characteristic lines in this spectrum. The green curves in Fig. 1 show a simulation of the spectrometer marking possible locations of events for different molecules. The breakup of helium dimers is clearly visible, and in addition lines from the residual gas can be identified.

This allows us to separate reactions of dimers and ionization processes in monomers (helium atoms). Furthermore, we can exclude background and possible reactions with larger clusters by selecting only events where both ions are emitted back to back. The rate of these events was about $7 \mathrm{~Hz}$ at a total ion rate of about $10 \mathrm{kHz}$, belonging mainly to photo-ionized helium monomers. The discrepancy between the rate of the detected dimers and the dimer fraction in the gas jet is due to different ionization mechanisms for dimers and monomers. In addition, the efficiency to detect the two ions of a dimer breakup in coincidence is decreased, compared with the efficiency to detect a single ion (from a monomer).

\section{RESULTS AND DISCUSSION}

In our measurement, we obtain the energy of all detected fragments of each reaction in coincidence. Figure 2 shows the kinetic energy of all measured electrons as a function of the KER at a photon energy of $68.86 \mathrm{eV}$. Here one can see two separated features, which can be associated to electrons from the two different ionization steps. The first group of electrons has a fixed energy of $3.46 \mathrm{eV}$, independent of the KER. These electrons originate from a photoionization process, where photon absorption leads to the ejection of one electron (photoelectron), while the ion remains in an excited state [see Eq. (1)]. The energy of the ejected photoelectrons is

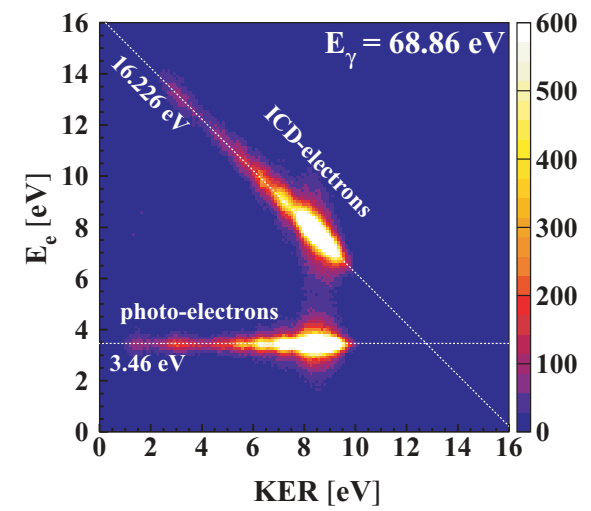

FIG. 2. (Color online) The kinetic energy of measured electrons as a function of the KER at a photon energy of $68.86 \mathrm{eV}$. This spectra allows a clear separation of ICD electrons and photoelectrons.

thereby given by $E_{\text {ephoto }}(n)=E_{\gamma}-24.59 \mathrm{eV}-(54.42 \mathrm{eV}-$ $\left.13.6 \mathrm{eV} \times \frac{4}{n^{2}}\right)$.

The diagonal feature in Fig. 2 can be identified as ICD electrons. They are emitted from the neutral center after relaxation of the excited dimer ion $\left(\mathrm{He}_{2}{ }^{+*}\right)$. The energy transferred is given by $E(n)=54.42 \mathrm{eV}-13.6 \mathrm{eV} \times \frac{4}{n^{2}}$. This energy enables the ionization of the neutral center in the dimer ion, while the rest $(16.226 \mathrm{eV}$ for $n=2)$ is converted into kinetic energy of the ICD electron and the KER. The constant sum of these two quantities leads to the observed diagonal in Fig. 2.

\section{A. Photoelectron angular distributions}

The clear signature for photoelectrons and ICD electrons allows us to analyze both species separately. For the photoelectron we measured the angle $\Theta$ between the direction of electron and the polarization vector. Figure 3 shows the distribution of $\Theta$ for all photoelectrons at a photon energy of $68.86 \mathrm{eV}$.

In a first approximation, we can compare photoionization of an atom of the dimer compound with the well-known process

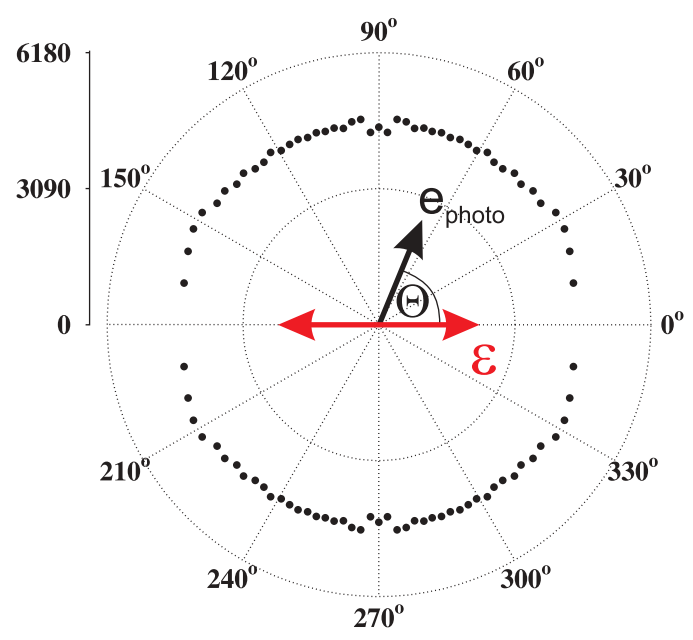

FIG. 3. (Color online) Angular distribution of photoelectron with respect to the polarization vector $\epsilon$ at a photon energy of $68.86 \mathrm{eV}$. The data are integrated over all KERs, and data points between $0^{\circ}$ and $90^{\circ}$ are mirrored into the other three quadrants. 


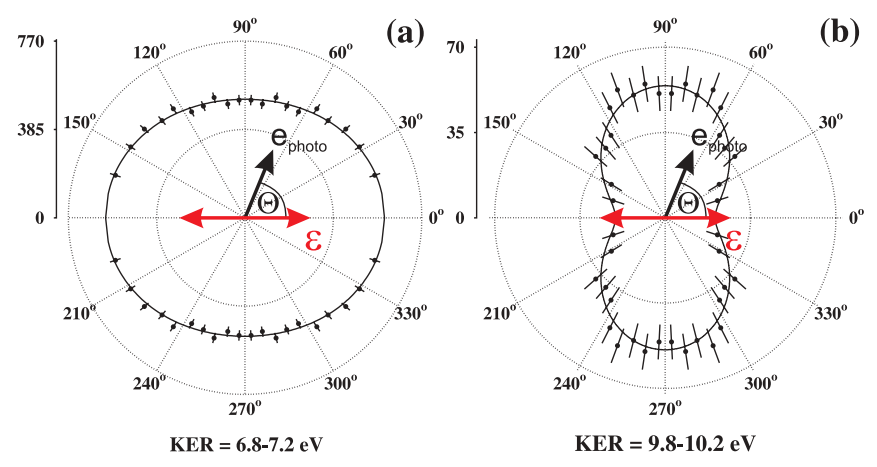

FIG. 4. (Color online) Photoelectron angular distribution with respect to the polarization axis at a photon energy of $68.86 \mathrm{eV}$ for two different regions of KER. (A subset of the data shown in Fig. 3. Data points between $0^{\circ}$ and $90^{\circ}$ are mirrored into the other three quadrants.) The solid line shows a fit of Eq. (3) to the data, yielding $\beta$ of +0.11 (a) and $\beta$ of -0.52 (b).

for a single helium atom. The differential cross section for each excited state can be described by

$$
\frac{d \sigma(h v, \Theta)}{d \Omega}(\Theta)=\frac{\sigma}{4 \pi}\left[1+\beta(h v) P_{2}(\cos \Theta)\right],
$$

where $\sigma(h v)$ is the total cross section, $P_{2}(\cos \Theta)$ is the second Legendre polynomial, and $\beta(h v)$ is the asymmetry parameter, which completely describes the shape of the angular distribution. Previous experiments with helium atoms showed an asymmetry parameter $\beta$ of 0.09 at the present photon energy [23]. This is already in good agreement with the almost isotropic distribution shown in Fig. 3. In Fig. 4, we plotted the distribution of $\Theta$ for different regions of the KER. A significant change with KER is observable.

These data sets can now be fitted using Eq. (3) to obtain $\beta$ as a function of the KER in steps of $0.5 \mathrm{eV}$ (see Fig. 5). The $\beta$ is very close to the atomic value up to a KER of $8 \mathrm{eV}$ and shows a sharp decrease above that energy. This effect can be explained by different populated states in the excited dimer ion $\mathrm{He}_{2}{ }^{+*}$.

A photon energy of $68.86 \mathrm{eV}$ allows single ionization plus excitation of a helium atom to $2 s$ or $2 p$ states only. Since the energy splitting between both states is only about $58 \mu \mathrm{eV}$ [24], the measured $\beta$ in previous experiments is a mixture of both states. Photoelectrons from $2 s$ excitations have $\beta(2 s)=2$. Hence only the fraction of populated $2 p$ states affects the measured asymmetry parameter. A theory of Jacobs $e$ t al. predicts $\beta(2 p)=-0.72$ (dashed line in Fig. 5) in the region of photon energies [25] considered in our measurement. This leads to the constant overall $\beta$ of 0.09 (dotted line in Fig. 5), which has been measured by Lindle et al. for the atomic case.

The observed KER dependence of $\beta$ for the dimer thus shows that the $s$ to $p$ ratio changes as a function of the KER. This is in line with the theory of Sisourat et al. [11] shown in Fig. 5. Depending on the populated atomic orbital, a combined system with molecular symmetry $\Sigma$ or $\Pi$ can be built. As $2 s$ and $2 p$ are very close in energy, nonadiabatic coupling can occur between states with the same molecular symmetry. Thus transitions between $\Sigma(2 s)$ and $\Sigma(2 p)$ states are possible. The upper panel in Fig. 5 shows the calculated contribution of states with the two symmetries to the total KER. The region

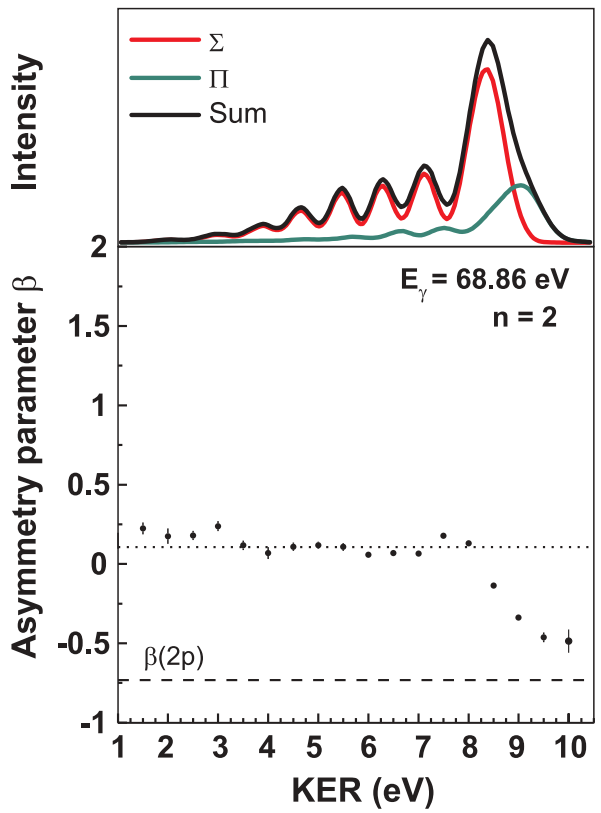

FIG. 5. (Color online) Top: calculated kinetic energy distribution for states of $\Sigma$ and $\Pi$ symmetry (from [11]). Bottom: Asymmetry parameter $\beta$ of the photoelectron as function of the KER. The dotted line indicates a measurement for helium atoms by Lindle et al. [23]. The dashed line shows a theory of $\beta$ for $2 p$ states only [25].

up to $8 \mathrm{eV}$ KER is dominated by $\Sigma$ states. Hence, $2 s$ as well as $2 p$ excitations can contribute to this region. In contrast, the KER above $9 \mathrm{eV}$ is dominated by excitations with a molecular symmetry of $\Pi$. These states can be constructed only from atomic excitations to $2 p$ orbitals. Accordingly, an increased fraction of $2 p$ states is expected for the region of high KER. The observed decrease of $\beta$ toward the value for pure $2 p$ excitation is in good agreement with these results.

\section{B. ICD electron angular distributions}

By measuring the complete momentum vector of all particles, we obtain the angle $\vartheta$ between the emission direction of an electron and the fragmentation direction of the dimer, that is, the direction of emission of the two He ions. For the photoelectrons, the fragmentation direction is irrelevant, since the dimer has time to rotate between the first ionization step and ICD. A semiclassical estimation leads to rotation periods of several picoseconds. Thus, they are of the same order as the lifetime of the excited system [11]. This will wash out any structure of the photoelectron angular distribution [26]. For the ICD electron, however, the fragmentation direction corresponds to the orientation of the dimer axis at the instant of ICD. Therefore, we are able to obtain ICD electron angular distributions in the body fixed frame of the dimer. Figure 6 shows this distribution integrated over all values of the KER.

We now investigate the angular distributions of the ICD electron for different regions of KERs (Fig. 7). A strong variation of $\vartheta$ with the KER is found.

For Auger electron angular distributions, at least three distinct physical effects determine the angular distribution [27,28]: (a) the shape of the bound-state orbitals (hole state and both participating electrons) [29,30], (b) multiple scattering of 


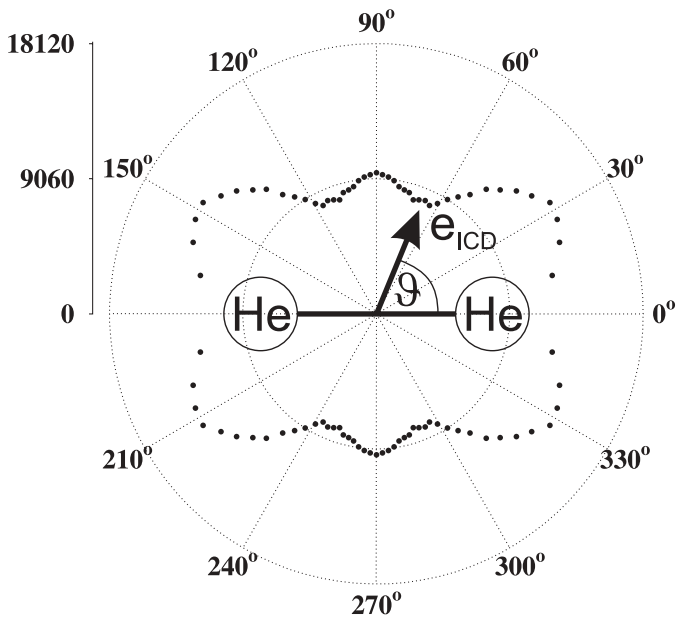

FIG. 6. Distribution of the angle $\vartheta$ between the dimer axis and the emission direction of the ICD electrons. (Distribution is integrated over all values of KER. Data points between $0^{\circ}$ and $90^{\circ}$ are mirrored into the other three quadrants.)

the ejected electron in the molecular potential [27,31], and (c) for homonuclear diatomics also two-center interference, resulting from the indistinguishability of the two ends of the molecule $[32,33]$. For ICD, we expect the same effects to play a role.

The variations with KER observed in Fig. 7 therefore clearly indicate that the dominating states in excited dimer ion $\mathrm{He}_{2}{ }^{+*}$ differ for different values of the KER. The ICD process with excitation to $n=2$ in the helium dimer is dominated by the population of four potential-energy curves [11]. As the final state of the $\mathrm{He}^{+}-\mathrm{He}^{+}$system allows for two different orientations of the spins, the process is composed of eight different transitions. Depending on the internuclear distance, these transitions contribute with different intensities.

Because no calculations of the angular distributions are currently available, we compare our data to the predictions of a very simple model. Our model has two major ingredients, which we believe capture most of the physics essence of ICD in the helium dimer. First, it is the analogy to photoionization due to the virtual photon exchange, and second, the two-center nature of the emission which leads to a distance- and wavelengthdependent interference term [32,34-36]. However, the model completely neglects multiple scattering of the ICD electron in the final two-center potential.

For dipole allowed transitions, ICD may be thought of as a photoionization of a neutral He atom by a (virtual) photon, emitted by the relaxation from $\mathrm{He}^{+}(2 p)$ to the ground state. The angular part of the wave function, emitted from one or the other center, is hence given by a spherical harmonic $Y_{l m}$. For ICD, the value of $l$ is restricted to 1 , while the $m$ value is the $m$ of the decaying neighbor. Following the suggestion of Cohen and Fano [34-36], the two-center nature of the problem can be approximately accounted for by coherently superimposing two spherical waves $e^{i \vec{k} \cdot \vec{r}}$ in a distance $\vec{R}$. In this approximation, the final ICD wave function is therefore of the form

$$
e^{i \vec{k} \cdot(\vec{r}-\vec{R} / 2)} Y_{l m} \pm e^{i \vec{k} \cdot(\vec{r}+\vec{R} / 2)} Y_{l m} .
$$
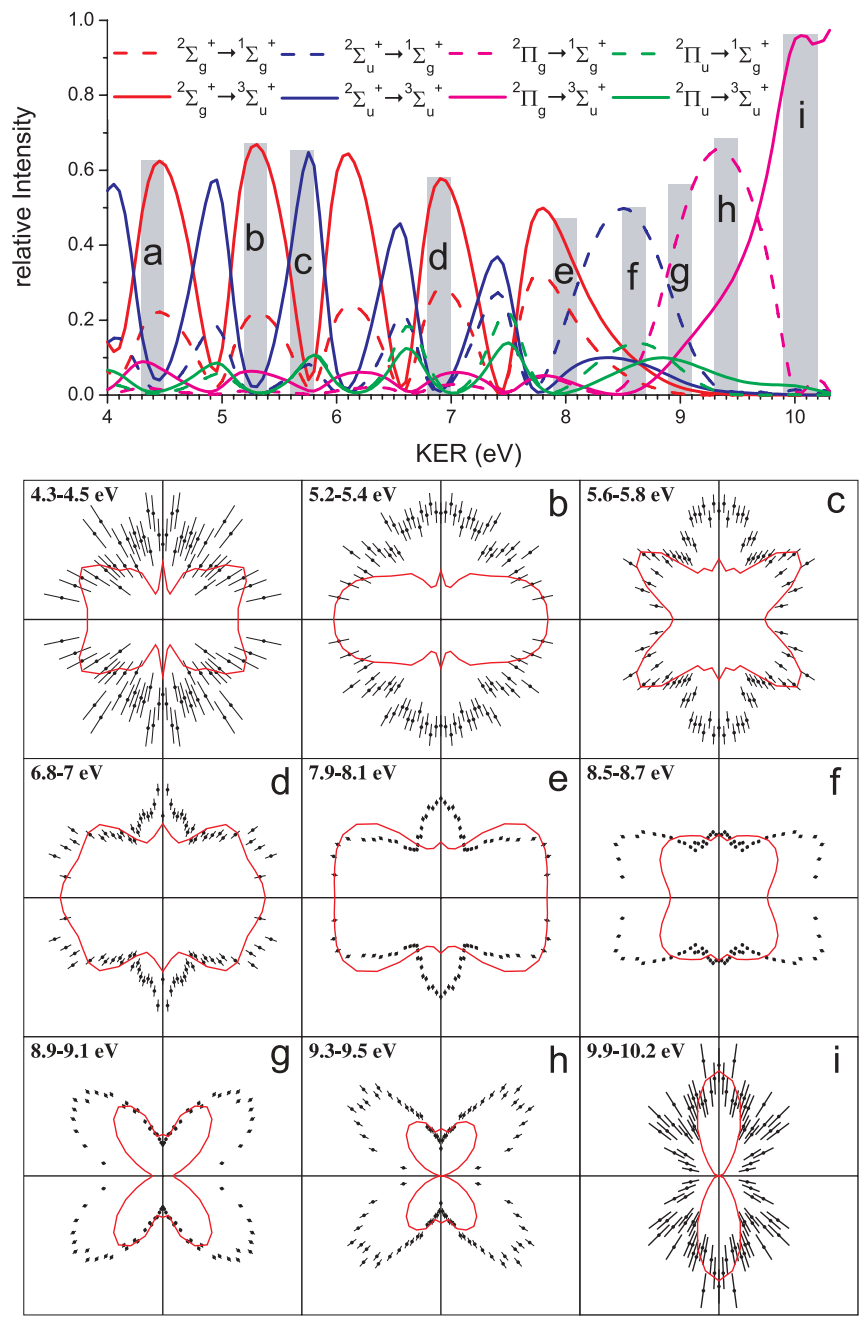

FIG. 7. (Color online) Angular distributions of the ICD electron with respect to the dimer axis (horizontal) for different regions of KER. (Datapoints between $0^{\circ}$ and $90^{\circ}$ are mirrored into the other three quadrants.) The continuous red lines represent a simple model. The upper panel shows the relative contributions of different transitions to the KER [11]. Selected areas are marked in this plot as the gray bars $(a-i)$.

Here $\vec{k}$ is the ICD electron momentum, $\vec{r}$ is its position, and $\vec{R}$ is the internuclear vector. The \pm together with the $m$ defines the parity of the ICD electron wave. It is given by the difference of the parity of the initial $\mathrm{He}_{2}{ }^{+*}$ and the final $\mathrm{He}_{2}{ }^{2+}$ molecular state. This yields to the following table:

\begin{tabular}{lc} 
Transition & $\psi(\vec{r}, \vec{k}, \vartheta, \vec{R})$ \\
\hline${ }^{2} \Sigma_{g}^{+} \rightarrow{ }^{1} \Sigma_{g}^{+}$ & $\cos (\vartheta) e^{i \vec{k} \cdot(\vec{r}-\vec{R} / 2)}+\cos (\vartheta) e^{i \vec{k} \cdot(\vec{r}+\vec{R} / 2)}$ \\
${ }^{2} \Sigma_{u}^{+} \rightarrow{ }^{3} \Sigma_{u}^{+}$ & \\
${ }^{2} \Sigma_{g}^{+} \rightarrow{ }^{3} \Sigma_{u}^{+}$ & $\cos (\vartheta) e^{i \vec{k} \cdot(\vec{r}-\vec{R} / 2)}-\cos (\vartheta) e^{i \vec{k} \cdot(\vec{r}+\vec{R} / 2)}$ \\
${ }^{2} \Sigma_{u}^{+} \rightarrow{ }^{1} \Sigma_{g}^{+}$ & \\
${ }^{2} \Pi_{g} \rightarrow{ }^{1} \Sigma_{g}^{+}$ & $\sin (\vartheta) e^{i \vec{k} \cdot(\vec{r}-\vec{R} / 2)}-\sin (\vartheta) e^{i \vec{k} \cdot(\vec{r}+\vec{R} / 2)}$ \\
${ }^{2} \Pi_{u} \rightarrow{ }^{3} \Sigma_{u}^{+}$ & \\
${ }^{2} \Pi_{g} \rightarrow{ }^{3} \Sigma_{u}^{+}$ & $\cos (\vartheta) e e^{i \vec{k} \cdot(\vec{r}-\vec{R} / 2)}+\cos (\vartheta) e^{i \vec{k} \cdot(\vec{r}+\vec{R} / 2)}$ \\
${ }^{2} \Pi_{u} \rightarrow{ }^{1} \Sigma_{g}^{+}$ & \\
\hline
\end{tabular}


In order to go beyond the reflection approximation (that was showed to be rather poor for the helium dimer in [12]), we computed as in [11] the vibrational levels of the neutral $\mathrm{He}_{2}, \mathrm{He}-\mathrm{He}^{+*}$ as well as the dissociative states of $\mathrm{He}^{+}-\mathrm{He}^{+}$. Incorporating the ICD electron wave function of our simple model in the transition amplitudes, the angular distribution at a fixed KER is given by

$$
\begin{aligned}
& \sigma\left(\theta,(\mathrm{KER})=E_{f}\right) \\
& \quad=\int d E_{e}\left|\sum_{j=1}^{n_{d}} \frac{\left\langle F_{f} \times \psi(\vec{r}, \vec{k}, \theta, \vec{R})|\hat{W}| D_{j}\right)\left(D_{j}|I\rangle\right.}{E_{e}+E_{f}-\epsilon_{j}+i \Gamma_{j} / 2}\right|^{2},
\end{aligned}
$$

where $E_{e}, E_{f}$, and $\epsilon_{j}+i \Gamma_{j} / 2$ are the energies of the ICD electron, of the final state $\left|F_{f}\right\rangle$, and of the decaying state $\left.\mid D_{j}\right)$, respectively. The states $|I\rangle$ and $\left|F_{f}\right\rangle$ are the nuclear eigenstates of the neutral $\mathrm{He}-\mathrm{He}$ and the final $\mathrm{He}^{+}-\mathrm{He}^{+}$ electronic states, respectively. The states $\left.\mid D_{j}\right)$ are the complex eigenstates of the electronic decaying state. The numbers $n_{d}$ of decaying nuclear eigenstates were chosen to ensure convergence of the results. The operator $\hat{W}$ is equal to $\sqrt{2 \pi \gamma_{D}}$, where $\gamma_{D}$ is the partial ICD rate, and $\psi(\vec{r}, \vec{k}, \theta, \vec{R})$ is defined in the previous table. The angular distribution for each of the eight ICD channels were computed and summed incoherently. The results are shown in Fig. 7.

Given the simplicity of the model, the agreement between the measured distribution and simulation is surprisingly good in the range of high KER and accordingly small internuclear distance, but the simulation fails at lower KER.

\section{CONCLUSION}

In conclusion, we have shown that the emission direction of photoelectrons in the laboratory frame strongly depends on the KER. As reason for this variation, we could identify a different ratio of excitations to $2 s$ and $2 p$ states. Thus, a separation between both atomic excitations is possible, if the atom is part of a dimer compound. An analysis of angular distributions of ICD electrons in the dimer fixed frame shows a strong dependency on the KER, as well. The rich structure observed calls for theoretical work on ICD electron angular distributions. Such molecular continuum electron angular distributions can be calculated very precisely if the molecular states involved are known. Therefore, the comparison of the experimental and calculated angular distribution can in turn be used to identify the molecular states involved [29,30]. We believe that this is a promising route to clarify the remaining differences in the experimentally obtained KER, electron energy distribution [10], and most advanced ICD calculations $[11,37]$.

\section{ACKNOWLEDGMENTS}

We thank the staff at BESSY for experimental support. This work was supported by the Koselleck Programm of DFG, and R.E.G. acknowledges support by Helmholtz Society, Grant VH-NG-331. We are grateful to L. S. Cederbaum, N. Kryzhevoi, and $\mathrm{Ph}$. Demekhin for stimulating discussions.
[1] L. S. Cederbaum, J. Zobeley, and F. Tarantelli, Phys. Rev. Lett. 79, 4778 (1997).

[2] T. Jahnke et al., Phys. Rev. Lett. 93, 163401 (2004).

[3] Y. Morishita et al., Phys. Rev. Lett. 96, 243402 (2006).

[4] P. Lablanquie, T. Aoto, Y. Hikosaka, Y. Morioka, F. Penent, and K. Ito, J. Chem. Phys. 127, 154323 (2007).

[5] S. Marburger, O. Kugeler, U. Hergenhahn, and T. Möller, Phys. Rev. Lett. 90, 203401 (2003).

[6] T. Jahnke et al., Nature Phys. 6, 139 (2010).

[7] M. Mucke, M. Braune, S. Barth, M. Förstel, T. Lischke, V. Ulrich, T. Arion, U. Becker, A. Bradshaw, and U. Hergenhahn, Nature Phys. 6, 143 (2010).

[8] V. Averbukh, I. B. Müller, and L. S. Cederbaum, Phys. Rev. Lett. 93, 263002 (2004).

[9] T. Jahnke et al., Phys. Rev. Lett. 99, 153401 (2007).

[10] T. Havermeier et al., Phys. Rev. Lett. 104, 133401 (2010).

[11] N. Sisourat, N. V. Kryzhevoi, P. Kolorenč, S. Scheit, T. Jahnke, and L. S. Cederbaum, Nature Phys. 6, 508 (2010).

[12] T. Havermeier et al., Phys. Rev. Lett. 104, 153401 (2010).

[13] T. Jahnke et al., J. Phys. B 40, 2597 (2007).

[14] K. Kreidi et al., J. Phys. B 41, 101002 (2008).

[15] K. Kreidi et al., Phys. Rev. A 78, 043422 (2008).

[16] K. Kreidi et al., Phys. Rev. Lett. 103, 033001 (2009).

[17] R. Dorner, V. Mergel, O. Jagutzki, L. Spielberger, J. Ullrich, R. Moshammer, and H. Schmidt-Bocking, Phys. Rep. 330, 95 (2000).

[18] J. Ullrich, R. Moshammer, A. Dorn, R. Dörner, L. Ph. H. Schmidt, and H. Schmidt-Böcking, Rep. Prog. Phys. 66, 1463 (2003).
[19] T. Jahnke, Th. Weber, T. Osipov, A. L. Landers, O. Jagutzki, L. Ph. H. Schmidt, C. L. Cocke, M. H. Prior, H. SchmidtBöcking, and R. Dörner, J. Electron Spectrosc. Relat. Phenom. 141, 229 (2004).

[20] W. Schöllkopf and J. P. Toennies, Science 266, 1345 (1994).

[21] R. E. Grisenti, W. Schöllkopf, J. P. Toennies, G. C. Hegerfeldt, T. Köhler, and M. Stoll, Phys. Rev. Lett. 85, 2284 (2000).

[22] O. Jagutzki, V. Mergel, K. Ullmann-Pfleger, L. Spielberger, U. Spillmann, R. Dörner, and H. Schmidt-Böcking, Nucl. Instrum. Methods Phys. Res., Sect. A 477, 244 (2002).

[23] D. W. Lindle, P. A. Heimann, T. A. Ferrett, and D. A. Shirley, Phys. Rev. A 35, 1128 (1987).

[24] G. W. F. Drake and W. C. Martin, Can. J. Phys. 76, 679 (1998).

[25] V. L. Jacobs and P. G. Burke, J. Phys. B 5, L67 (1972).

[26] Th. Weber et al., J. Phys. B 34, 3669 (2001).

[27] K. Zähringer, H. D. Meyer, and L. S. Cederbaum, Phys. Rev. A 46, 5643 (1992).

[28] D. Dill, J. R. Swanson, S. Wallace, and J. L. Dehmer, Phys. Rev. Lett. 45, 1393 (1980).

[29] N. A. Cherepkov et al., Phys. Rev. A 80, 051404(R) (2009).

[30] S. K. Semenov et al., Phys. Rev. A 81, 043426 (2010).

[31] C. S. Fadley, Surf. Sci. Rep. 19, 231 (1993).

[32] N. A. Cherepkov et al., Phys. Rev. A 82, 023420 (2010).

[33] M. S. Schöffler et al., Science 320, 920 (2008).

[34] H. D. Cohen and U. Fano, Phys. Rev. 150, 30 (1966).

[35] J. Fernández, O. Fojón, A. Palacios, and F. Martín, Phys. Rev. Lett. 98, 043005 (2007).

[36] D. Akoury et al., Science 318, 949 (2007).

[37] P. Kolorenč, N. V. Kryzhevoi, N. Sisourat, and L. S. Cederbaum, Phys. Rev. A 82, 013422 (2010). 